\title{
Erratum
}

\section{A simple and disposable sweat collector}

\author{
G. R. Brisson ${ }^{1}$, P. Boisvert ${ }^{2}$, F. Perronet ${ }^{2}$, H. Perrault ${ }^{3}$, D. Boisvert ${ }^{1}$, and J. S. Lafond ${ }^{4}$ \\ ${ }^{1}$ Institut National de la Recherche Scientifique (INRS-Santé), \\ ${ }^{2}$ Université de Montréal, \\ ${ }^{3}$ McGill University, \\ ${ }^{4}$ Université du Québec à Montréal, Canada
}

Eur J Appl Physiol (1991) 63:269-272

The following replaces this sub-section given on $\mathrm{p} 270$ of the Methods:

Fractional sampling. When serial samples are collected, one can calculate both the sweat rate and the concentration of any component. For sweat rate, one must first calculate the volume of encapsulated liquid $\left(V_{n}\right)$ just before sampling:

$V_{n}=\frac{c_{n-1}\left(V_{n-1}-S_{n-1}\right)}{c_{n}}$

where $V_{n}$ is the volume in the capsule prior to the $n$th sample, $c_{n}$ is the concentration of the marker in the $n$th sample, and $S_{n-1}$ is the volume of the " $n-1$ " sample. Then, the volume of sweat excreted $\left(V_{S n}\right)$ into the capsule between two successive samplings $(n-1, n)$ can be calculated:

$V_{S n}=V_{n}-V_{n-1}+S_{n-1}$

To assess component concentration in sweat volume $\left(c_{S n}\right)$, one must first calculate the amount of component $\left(Q_{n}\right)$ contained in the $n$th sample:

$Q_{n}=c_{A n} \cdot V_{n}$ where $c_{A n}$ is the component concentration in the sweat sample.

Thereafter, the amount of component excreted $\left(Q_{S n}\right)$ in the capsule between two consecutive samples can be calculated:

$Q_{S n}=\left(c_{A n} \cdot V_{n}\right)-c_{A n-1}\left(V_{n-1}-S_{n-1}\right)$

Hence, the component concentration in sweat volume excreted $\left(c_{S n}\right)$ is:

$c_{S n}=\frac{Q_{S n}}{V_{S n}}$

If the vacutainer aspirating system is used and the sweat volume is very low, the equation given below can be used:

$c_{S}=\frac{Q_{S}}{V_{S}}=\frac{c_{S e} \cdot V_{c}}{V_{c}-V_{i}}$

where $c_{S e}$ is the component concentration measured in the sample collected at the end of the observation period; $V_{c}$ is the volume of sweat measured at the end of the observation period and corrected for aspiration volume losses (approximately $200 \mu \mathrm{l}$ for a vacutainer aspirating system from a initially dry capsule); and $V_{i}$ is the volume of deionized water added to the capsule. 